\title{
POSITIVE SOLUTIONS IN AN ANNULUS FOR NONLINEAR DIFFERENTIAL EQUATIONS ON A MEASURE UIATN
}

\author{
CHUAN JEN CHYAN, JOHNNY HENDERSON AND HUI CHUN LO
}

\begin{abstract}
We study the existence of positive solutions of the second order differential equation in an annulus on a measure chain, $u^{\Delta \Delta}(t)+f(u(\sigma(t)))=0, t \in[0,1]$, satisfying the boundary conditions, $\alpha y(0)-\beta y^{\Delta}(0)=0$ and $\gamma y(\sigma(1))+\delta y^{\Delta}(\sigma(1))=0$, where $f$ is a positive function and $f(x)$ is sublinear (respectively superlinear) at $x=0$ and is superlinear (respectively sublinear) at $x=\infty$. The methods involve applications of a fixed point theorem for operators on a cone in a Banach space.
\end{abstract}

\section{Introduction}

In this paper, we study the existence of positive solutions of a certain second order differential equation in an annulus on a measure chain. Much recent attention has been given to differential equations on measure chains, and we refer the reader to [4], [9] and [15] for some historical works as well as to the more recent papers [1], [1], [2], [5], [6], [10], [11] and the book [19] for excellent references on these types of equations. Before introducing the problems of interest for this paper, we present some definitions and notation which are common to the recent literature. Our sources for this background material are the two papers by Erbe and Peterson [10], [11].

Definitions 1.1. Let $T$ be a closed subset of $R$, and let $T$ have the subspace topology inherited from the Euclidean topology on $R$. For $t<\sup T$ and $r>\inf T$, define the forward jump operator, $\sigma$, and the backward jump operator, $\rho$, respectively, by

$$
\begin{aligned}
& \sigma(t)=\inf \{\tau \in T \mid \tau>t\} \in T, \\
& \rho(r)=\sup \{\tau \in T \mid \tau<r\} \in T,
\end{aligned}
$$

for all $t, r \in T$. If $\sigma(t)>t, t$ is said to be right scattered, and if $\rho(r)<r, r$ is said to be left scattered. If $\sigma(t)=t, t$ is said to be right dense, and if $\rho(r)=r, r$ is said to be left dense.

Received March 22, 1999; revised May 14, 1999.

1991 Mathematics Subject Classification. 34B99, 39A99

Key words and phrases. Differential equation, measure chain, boundary value problem. 
Definitions 1.2. For $x: T \rightarrow R$ and $t \in T$ (if $t=\sup T$, assume $t$ is not left scattered), define the delta derivative of $x(t), x^{\Delta}(t)$, to be the number (when it exists), with the property that, for any $\epsilon>0$, there is a neighborhood, $U$, of $t$ such that

$$
\left|[x(\sigma(t))-x(s)]-x^{\Delta}(t)[\sigma(t)-s]\right| \leq \epsilon|\sigma(t)-s|
$$

for all $s \in U$. The second delta derivative of $x(t)$ is defined by $x^{\Delta \Delta}(t)=\left(x^{\Delta}\right)^{\Delta}(t)$.

If $F^{\Delta}(t)=h(t)$, then define the integral by

$$
\int_{a}^{t} h(s) \Delta s=F(t)-F(a)
$$

Throughout this paper, we assume $T$ is a closed subset of $R$ with $0,1 \in T$.

Definition 1.3. Define the closed interval, $[0,1]$, in $T$ by

$$
[0,1]=\{t \in T \mid 0 \leq t \leq 1\}
$$

Other closed, open, and half-open intervals in $T$ are similarly defined.

In this paper, we consider the existence of positive solutions of the differential equation on a measure chain,

$$
u^{\Delta \Delta}(t)+f(u(\sigma(t)))=0, \quad t \in[0,1],
$$

satisfying

$$
\alpha y(0)-\beta y^{\Delta}(0)=0 \quad \gamma y(\sigma(1))+\delta y^{\Delta}(\sigma(1))=0,
$$

where $f: R^{+} \rightarrow R^{+}$is continuous and $d=\gamma \beta+\alpha \delta+\alpha \gamma(\sigma(1))>0$.

We remark that by a solution, $u$, of (1), (2), we mean $u:\left[0, \sigma^{2}(1)\right] \rightarrow R, u$ satisfies (1) on $[0,1]$, and $u$ satisfies the boundary conditions (2).

This paper constitutes an extension of the recent work by Erbe and Peterson [11] in which they obtained positive solutions of both (1), (2) for the cases when either $f(x)$ is superlinear (at $x=0$ and $x=\infty$ ), or $f(x)$ is sublinear (at $x=0$ and $x=\infty$ ). The solutions obtained in [11] were found to belong to the intersection of a cone with an annular type region. In a more recent paper, Erbe and Peterson [12] applied index theory in a cone to obtain multiple positive solutions of (1), (2). Our goal is to extend to differential equations on measure chains the works of Erbe, $\mathrm{Hu}$, and Wang [13], Erbe and Tang [14], and Kaufmann [17], to obtain two positive solutions of (1), (2), when $f$ is superlinear at one endpoint (zero or infinity) and sublinear at the other (respectively, infinity or zero); that is when either $(i) f_{0}=\infty$ and $f_{\infty}=\infty$, or $(i i) f_{0}=0$ and $f_{\infty}=0$, where,

$$
f_{0}=\lim _{x \rightarrow 0^{+}} \frac{f(x)}{x} \quad \text { and } \quad f_{\infty}=\lim _{x \rightarrow+\infty} \frac{f(x)}{x} .
$$

We will make our assumptions on $f$ more precise in Section 3 . The results herein are also related to those by Atici [3] and Eloe and Henderson [8]. 
A Krasnosel'skii fixed point theorem [20] is applied to yield positive solutions of (1), (2) in our arguments. These methods have been used successfully, in the cases when (1) is either an ordinary differential equation or a finite difference equation; see [17], [18], $[22]$.

In Section 2, we present some properties of a Green's function associated with (1), (2) that are used in defining a positive operator. We also state the Krasnosel'skii fixed point theorem. In Section 3, we give an appropriate Banach space and construct a cone on which we apply the fixed point theorem yielding twin positive solutions of (1), (2).

\section{Some Preliminaries}

In this section, we state the above mentioned Krasnosel'skii fixed point theorem. We will apply this fixed point theorem in the next section to a completely continuous integral operator whose kernel, $G(t, s)$, is the Green's function for

$$
\begin{gathered}
-y^{\Delta \Delta}(t)=0, \\
\alpha y(0)-\beta y^{\Delta}(0)=0 \quad \gamma y(\sigma(1))+\delta y^{\Delta}(\sigma(1))=0 .
\end{gathered}
$$

Erbe and Peterson [11] have found

$$
G(t, s)=\left\{\begin{array}{l}
\frac{1}{d}\{\alpha t+\beta\}\{\gamma(\sigma(1)-\sigma(s))+\delta\}, t \leq s \\
\frac{1}{d}\{\alpha \sigma(s)+\beta\}\{\gamma(\sigma(1)-t)+\delta\}, \sigma(s) \leq t
\end{array}\right.
$$

where

from which

$$
d=\gamma \beta+\alpha \delta+\alpha \gamma(\sigma(1))>0
$$

$$
\begin{aligned}
& G(t, s)>0, \quad(t, s) \in(0, \sigma(1)) \times(0,1), \\
& \frac{G(t, s)}{G(\sigma(s), s)}= \begin{cases}\frac{\alpha t+\beta}{\alpha \sigma(s)+\beta}, & t \leq s, \\
\frac{\gamma(\sigma(1)-t)+\delta}{\gamma(\sigma(1)-\sigma(s))+\delta}, & \sigma(s) \leq t .\end{cases}
\end{aligned}
$$

This implies

$$
G(t, s) \leq G(\sigma(s), s) \quad \text { for } \quad t \in[0, \sigma(1)], s \in[0,1],
$$

and it is also shown in [11] that

$$
G(t, s) \geq k G(\sigma(s), s), \quad t \in\left[\frac{\sigma(1)}{4}, \frac{3 \sigma(1)}{4}\right], s \in[0,1],
$$

where $k=\min \left\{\frac{\alpha \sigma(1)+4 \beta}{4 \alpha \sigma(1)+4 \beta}, \frac{\gamma \sigma(1)+4 \delta}{4 \gamma(\sigma(1)-\sigma(0))+4 \delta}\right\}$.

We will apply the following fixed point theorem which can be found in the book by Krasnosel'skii [20] as well as in the book by Deimling [7].

Theorem 2.1. Let $B$ be a Banach space, and let $P \subset B$ be a cone in $B$. Assume $\Omega_{1}, \Omega_{2}$ are open subsets of $B$ with $0 \in \Omega_{1} \subset \bar{\Omega}_{1} \subset \Omega_{2}$, and let

$$
T: P \cap\left(\bar{\Omega}_{2} \backslash \Omega_{1}\right) \rightarrow P
$$

be a completely continuous operator such that either, 
(i) $\|T u\| \leq\|u\|, u \in P \cap \partial \Omega_{1}$, and $\|T u\| \geq\|u\|, u \in P \cap \partial \Omega_{2}$, or

(ii) $\|T u\| \geq\|u\|, u \in P \cap \partial \Omega_{1}$, and $\|T u\| \leq\|u\|, u \in P \cap \partial \Omega_{2}$.

Then $T$ has a fixed point in $P \cap\left(\bar{\Omega}_{2} \backslash \Omega_{1}\right)$.

\section{Solutions of $(1.1),(1.2)$ in a Cone}

In this section, we apply Theorem 2.1 to the eigenvalue problem (1), (2). Throughout this section, we assume $\sigma(1)$ is right dense so that $G(t, s) \geq 0$, for $t \in\left[0, \sigma^{2}(1)\right], s \in$ $[0, \sigma(1)]$.

Assume also throughout this section that $[0, \sigma(1)]$ is such that

$$
\begin{aligned}
& \xi=\min \left\{t \in T \mid t \geq \frac{\sigma(1)}{4}\right\}, \text { and } \\
& \omega=\max \left\{t \in T \mid t \leq \frac{3 \sigma(1)}{4}\right\}
\end{aligned}
$$

both exist and satisfy,

$$
\frac{\sigma(1)}{4} \leq \xi<\omega \leq \frac{3 \sigma(1)}{4}
$$

and if $\sigma(\omega)=1$, also assume $\sigma(\omega)<\sigma(1)$. Next, let $\tau \in[\xi, \omega]$ be defined by

$$
\int_{\xi}^{\omega} G(\tau, s) \Delta s=\max _{t \in[\xi, \omega]} \int_{\xi}^{\omega} G(t, s) \Delta s .
$$

Finally, we define

$$
\ell=\min _{s \in[0, \sigma(1)]} \frac{G(\sigma(\omega), s)}{G(\sigma(s), s)}
$$

and set

$$
m=\min \{k, \ell\} .
$$

We note that $u(t)$ is a solution of (1), (2), if and only if,

$$
u(t)=\int_{0}^{\sigma(1)} G(t, s) f(u(\sigma(s))) \Delta s, \quad t \in\left[0, \sigma^{2}(1)\right] .
$$

For our constructions, let $B=\left\{x:\left[0, \sigma^{2}(1)\right] \rightarrow R \mid x\right.$ is bounded $\}$, with norm $\|x\|=$ $\sup \left\{|x(t)|: t \in\left[0, \sigma^{2}(1)\right]\right\}$. Then, define the cone $P \subset B$

$$
P=\left\{x \in B \mid x(t) \geq 0 \text { on }\left[0, \sigma^{2}(1)\right], \text { and } x(t) \geq m\|x\| \text {, for } t \in[\xi, \sigma(\omega)]\right\} .
$$

Theorem 3.1. Assume that $f$ satisfies the following conditions:

(A) $f_{0}=\infty$ and $f_{\infty}=\infty$. 
(B) There exists a $p>0$ such that, if $0 \leq x \leq p$, then,

$$
f(x) \leq \eta p
$$

where,

$$
\eta=\left(\int_{0}^{\sigma(1)} G(\sigma(s), s) \Delta s\right)^{-1}
$$

Then there exist at least two solutions $u_{1}, u_{2}$ of (1), (2) which belong to $P$, such that

$$
0 \leq\left\|u_{1}\right\| \leq p \leq\left\|u_{2}\right\| \text {. }
$$

Proof. Define an integral operator $T: P \rightarrow B$ by

$$
T u(t)=\int_{0}^{\sigma(1)} G(t, s) f(u(\sigma(s))) \Delta s, \quad u \in P .
$$

We seek a fixed point of $T$ which belongs to $P$, and so we first claim $T: P \rightarrow P$. We choose $u \in P$. Then from (7),

$$
\begin{aligned}
T u(t) & =\int_{0}^{\sigma(1)} G(t, s) f(u(\sigma(s))) \Delta s \\
& \leq \int_{0}^{\sigma(1)} G(\sigma(s), s) f(u(\sigma(s))) \Delta s,
\end{aligned}
$$

and so

$$
\|T u\| \leq \int_{0}^{\sigma(1)} G(\sigma(s), s) f(u(\sigma(s))) \Delta s .
$$

So, from (8) and (13), for $u \in P$,

$$
\begin{aligned}
\min _{t \in[\xi, \omega]} T u(t) & =\min _{t \in[\xi, \omega]} \int_{0}^{\sigma(1)} G(t, s) f(u(\sigma(s))) \Delta s \\
& \geq \int_{0}^{\sigma(1)} k G(\sigma(s), s) f(u(\sigma(s))) \Delta s \\
& \geq k\|T u\| \\
& \geq m\|T u\|,
\end{aligned}
$$

and from (10)

$$
\begin{aligned}
T u(\sigma(\omega)) & =\int_{0}^{\sigma(1)} G(\sigma(\omega), s) f(u(\sigma(s))) \Delta s \\
& \geq \int_{0}^{\sigma(1)} \ell G(\sigma(s), s) f(u(\sigma(s))) \Delta s \\
& \geq m \int_{0}^{\sigma(1)} G(\sigma(s), s) f(u(\sigma(s))) \Delta s \\
& \geq m\|T u\| .
\end{aligned}
$$


Thus $T u \in P$, and we conclude $T: P \rightarrow P$. The standard arguments show $T$ is completely continuous.

From $f_{0}=\infty$ there is an $p>r>0$ such that $f(u) \geq M u$, for $0 \leq u \leq r$, where the constant $M>0$ satisfies

$$
M m \int_{\xi}^{\omega} G(\tau, s) \Delta s \geq 1 .
$$

Let

$$
\Omega_{1}=\{x \in B \mid\|x\|<r\},
$$

and then choose $u \in P$ with $\|u\|=r$. Then

$$
\begin{aligned}
T u(\tau) & =\int_{0}^{\sigma(1)} G(\tau, s) f(u(\sigma(s))) \Delta s \\
& \geq \int_{\xi}^{\omega} G(\tau, s) f(u(\sigma(s))) \Delta s \\
& \geq M \int_{\xi}^{\omega} G(\tau, s) u(\sigma(s)) \Delta s \\
& \geq M m \int_{\xi}^{\omega} G(\tau, s) \Delta s\|u\| \\
& \geq\|u\| .
\end{aligned}
$$

Thus, $\|T u\| \geq\|u\|$, and in particular,

$$
\|T u\| \geq\|u\|, \text { for } u \in P \cap \partial \Omega_{1} .
$$

Now consider $u \in P$ with $\|u\|=p$. From condition (B),

$$
\begin{aligned}
T u(t) & =\int_{0}^{\sigma(1)} G(t, s) f(u(\sigma(s))) \Delta s \\
& \leq \int_{0}^{\sigma(1)} G(\sigma(s), s) f(u(\sigma(s))) \Delta s \\
& \leq \int_{0}^{\sigma(1)} G(\sigma(s), s) \Delta s p \eta \\
& =\|u\| .
\end{aligned}
$$

If we define $\Omega_{2}=\{u \in B:\|u\|<p\}$, then

$$
\|T u\| \leq\|u\|, \quad u \in P \cap \partial \Omega_{2} .
$$

Theorem 2.1, together with (15) and (16), implies that there exists a fixed point, $u_{1}$, satisfying $r<\left\|u_{1}\right\|<p$. 
Next, using condition (A) again, we know there exists an $H_{1}>0$ such that

$$
f(u) \geq \varepsilon u,
$$

for all $u \geq H_{1}$, where we choose $\varepsilon>0$ such that,

$$
\varepsilon m \int_{\xi}^{\omega} G(\tau, s) \Delta s \geq 1
$$

Let $H=\max \left\{2 p, \frac{H_{1}}{m}\right\}$ and pick $u \in P$ with $\|u\|=H$. Since $u(t) \geq m\|u\| \geq H_{1}$ on $t \in[\xi, \sigma(\omega)]$, we have

$$
\begin{aligned}
T u(\tau) & =\int_{0}^{\sigma(1)} G(\tau, s) f(u(\sigma(s))) \Delta s \\
& \geq \varepsilon \int_{\xi}^{\omega} G(\tau, s) u(\sigma(s)) \Delta s \\
& \geq \varepsilon m \int_{\xi}^{\omega} G(\tau, s) \Delta s\|u\| \\
& \geq\|u\| .
\end{aligned}
$$

Set $\Omega_{3}=\{u \in B:\|u\|<H\}$. Then

$$
\|T u\| \geq\|u\|, \quad u \in P \cap \partial \Omega_{3} .
$$

Theorem 2.1, together with (16) and (19), implies that there exists a fixed point, $u_{2}$, of $T$ such that $p<\left\|u_{2}\right\|<H$ and the proof is complete.

We now consider the case $f_{0}=0$ and $f_{\infty}=0$.

Theorem 3.2. Assume that $f$ satisfies the following conditions:

$\left(A^{\prime}\right) f_{0}=0$ and $f_{\infty}=0$.

$\left(B^{\prime}\right)$ There exists a $q>0$ such that, if $m q \leq x \leq q$, then,

$$
f(x) \geq \lambda q
$$

where,

$$
\lambda=\left(\int_{\xi}^{\omega} G(\tau, s) \Delta s\right)^{-1} .
$$

Then there exist at least two solutions $u_{1}, u_{2}$ of (1), (2) which belong to $P$, such that

$$
0 \leq\left\|u_{1}\right\| \leq q \leq\left\|u_{2}\right\|
$$

Proof. From $f_{0}=0$ there is an $0<r<q$ such that $f(u) \leq \eta u$ for $0 \leq u \leq r$ where the constant

$$
\eta=\left(\int_{0}^{\sigma(1)} G(\sigma(s), s) \Delta s\right)^{-1}
$$


Let

$$
\Omega_{1}=\{x \in B \mid\|x\|<r\}
$$

Then

$$
\begin{aligned}
T u(t) & =\int_{0}^{\sigma(1)} G(t, s) f(u(\sigma(s))) \Delta s \\
& \leq \int_{0}^{\sigma(1)} G(\sigma(s), s) \eta u(\sigma(s)) \Delta s \\
& \leq \eta \int_{0}^{\sigma(1)} G(\sigma(s), s) \Delta s\|u\| \\
& \leq\|u\| .
\end{aligned}
$$

If we define $\Omega_{1}=\{u \in B:\|u\|<r\}$, then

$$
\|T u\| \leq\|u\|, \quad u \in P \cap \partial \Omega_{1} .
$$

Now consider $u \in P$ with $\|u\|=q$. Then, from $\left(B^{\prime}\right)$,

$$
\begin{aligned}
T u(\tau) & =\int_{0}^{\sigma(1)} G(\tau, s) f(u(\sigma(s))) \Delta s \\
& \geq \int_{\xi}^{\omega} G(\tau, s) f(u(\sigma(s))) \Delta s \\
& \geq \lambda \int_{\xi}^{\omega} G(\tau, s) \Delta s q \\
& \geq\|u\| .
\end{aligned}
$$

Thus, $\|T u\| \geq\|u\|$, and in particular, if we define $\Omega_{2}=\{u \in B:\|u\|<q\}$, then

$$
\|T u\| \geq\|u\|, \text { for } u \in P \cap \partial \Omega_{2} .
$$

Theorem 2.1, together with (20) and (21), implies that there exists a fixed point, $u_{1}$, satisfying $r<\left\|u_{1}\right\|<q$. Returning to condition $\left(A^{\prime}\right)$, we know that for any $\varepsilon>0$, there exists an $M>0$ such that,

$$
f(u) \leq M+\varepsilon u \quad \text { for } \quad u \geq 0,0 \leq t \leq 1
$$

And so,

$$
\begin{aligned}
T u(t) & \leq \int_{0}^{\sigma(1)} G(t, s)[M+\varepsilon u(\sigma(s))] \Delta s \\
& \leq M \int_{0}^{\sigma(1)} G(\sigma(s), s) \Delta s+\varepsilon \int_{0}^{\sigma(1)} G(\sigma(s), s) u(\sigma(s)) \Delta s .
\end{aligned}
$$


We choose $\varepsilon>0$ sufficiently small and $L>\frac{M}{\eta}$ sufficiently large, so that for $u \in P \cap \partial \Omega_{3}$

$$
\|T u\| \leq L=\|u\|
$$

where

$$
\Omega_{3}=\{x \in B:\|u\|<L\} .
$$

We obtain the desired result $0 \leq\left\|u_{1}\right\| \leq q \leq\left\|u_{2}\right\|$ by appealing to (20), (21), (22) and Theorem 2.1. This completes the proof.

\section{Acknowledgement}

This paper is supported by the National Science Council of the Republic of China.

\section{References}

[1] R. P. Agarwal and M. Bohner, Basic calculus on time scales and some of its applications, preprint.

[2] R. P. Agarwal, M. Bohner and P. Wong, Sturm-Liouville eigenvalue problems on time scales, Appl. Math. Comput., in press.

[3] F. Atici, Two positive solutions of a boundary value problem for difference equations, J. Difference Eqns. Appl. 1(1995), 263-270.

[4] B. Aulback and S. Hilger, Linear dynamic processes with inhomogeneous time scale, Nonlinear Dynamics and Quantum Dynamical Systems, Math. Res. 59, Akademie Verlag, Berlin, 1990.

[5] C. J. Chyan and J. Henderson, Eigenvalue problems for nonlinear differential equations on a measure chain, J. Math. Anal. Appl., in press.

[6] C. J. Chyan, J. M. Davis, J. Henderson and W. K. C. Yin, Eigenvalue comparisons for nonlinear differential equations on a measure chain, Electronic Journal of Differential Equations 1998(1998), 1-7.

[7] K. Deimling, Nonlinear Functional Analysis, Springer-Verlag, New York, 1985.

[8] P. W. Eloe and J. Henderson, Positive solutions and nonlinear $(k, n-k)$ conjugate eigenvalue problems, Differential Equations Dynam. Systems 6(1998), 309-317.

[9] L. H. Erbe and S. Hilger, Sturmian theory on measure chains, Differential Equations Dynam. Systems 1(1993), 223-246.

[10] L. H. Erbe and A. Peterson, Green's functions and comparison theorems for differential equations on measure chains, Dynam. Contin. Discrete Impuls. Systems, in press.

[11] L. H. Erbe and A. Peterson, Positive solutions for a nonlinear differential equation on a measure chain, Math. Comput. Modelling, in press.

[12] L. H. Erbe and A. Peterson, Eigenvalue conditions and positive solutions, preprint.

[13] L. H. Erbe, S. Hu and H. Wang, Multiple positive solutions of some boundary value problems, J. Math. Anal. Appl. 184(1994), 640-648.

[14] L. H. Erbe and M Tang, Existence and multiplicity of positive solutions to nonlinear boundary value prob̆lems, Differential Equations Dynam. Systems 4(1996), 313-320.

[15] S. Hilger, Analysis on measure chains - a unified approach to continuous and discrete calculus, Resultate Math. 18(1990), 18-56. 
[16] J. Henderson and H. Wang, Positive solutions for nonlinear eigenvalue problems, J. Math. Anal. Appl. 208(1997), 252-259.

[17] E. R. Kaufmann, Multiple positive solutions for higher order boundary value problems, Rocky Mountain J. Math. 28(1998), 1017-1028.

[18] J. Henderson and E. R. Kaufmann, Multiple positive solutions for focal boundary value problems, Comm. Appl. Anal. 1(1997), 53-60.

[19] B. Kaymakcalan, V. Lakshmikantham and S. Sivasundaram, Dynamical Systems on Measure Chains, Kluwer Academic Publishers, Boston, 1996.

[20] M. A. Krasnosel'skii, Positive Solutions of Operator Equations, Noordhoff, Groningen, 1964.

[21] S. Lauer, Positive Solutions of Boundary Value Problems for Nonlinear Difference Equations, Ph. D. dissertation, Auburn University, 1997.

[22] H. Wang, On the existence of positive solutions for semilinear elliptic equations in the annulus, J. Diff. Eqns. 109(1994), 1-7.

Department of Mathematics, Tamkang University, Taipei, Taiwan, 251.

E-mail: chuanjen@mail.tku.edu.tw

Department of Mathematics, Auburn University, Auburn, Alabama 36849-5310 USA.

E-mail: hendej2@mail.auburn.edu

Department of Mathematics, Tamkang University, Taipei, Taiwan, 251.

E-mail: g7150044@tkgis.tku.edu.tw 